REVISTA DE CIENCIAS AGRÍCOLAS

Volumen 31 (1) : 78 - 91

Segundo Semestre

ISSN Impreso 0120-0135

\title{
USO E PERSPECTIVA DE Clonostachys rosea COMO AGENTE DE BIOCONTROLE
}

\section{USE AND PROSPECT OF Clonostachysroseaas A BIOCONTROL AGENT}

\author{
Rodrigo Moreira Saraiva ${ }^{1}$; Paulo Eduardo França de M. ${ }^{1}$; Álefe Vitorino Borges ${ }^{1}$; \\ Luiz Antonio Maffia ${ }^{1}$
}

Data de recepção: 5 de maio de 2014

Data de aceitação: 10 de junho de 2014

\begin{abstract}
RESUMO
O uso de métodos de controle de doenças de plantas mais sustentáveis é uma necessidade na agricultura atual. Nessa perspectiva, o controle biológico de doenças de plantas surge como uma alternativa eficaz e viável no contexto de manejo integrado. Dentre os vários agentes de biocontrole estudados, ênfase vem sendo dada ao fungo Clonostachys rosea por seu potencial de uso na agricultura. $\mathrm{O}$ fungo pode ser encontrado em diferentes regiões, colonizando todas as partes de diferentes espécies vegetais e, ainda, possui a capacidade de permanecer endofiticamente em algumas plantas. No que diz respeito ao controle biológico, C. rosea é comprovadamente eficiente no controle de diversos patógenos em uma ampla gama de hospedeiro. Além disso, há relatos do antagonista como promotor de crescimento e indutor de resistência em algumas espécies vegetais, e como fungo entomopatogênico. Pela eficiência e versatilidade do antagonista C. rosea, buscamos nessa revisão fornecer uma visão global sobre as perspectivas de uso do antagonista no contexto do controle biológico.
\end{abstract}

Palavras-chave: Controle biológico, antagonistas, fungos endofíticos, Bionectria ochroleuca.

1 Universidade Federal de Viçosa (UFV), Departamento de Fitopatologia 36570 - 000 Viçosa, MG, Brasil. rodrigo.msaraiva@gmail.com. 


\begin{abstract}
The use of more sustainable control methods for plant diseases is a necessity in today's agriculture. In this perspective, the biological control of plant diseases emerges as an effective and viable alternative in the context of integrated management. Among some biocontrol agents studied, emphasis has been given to the fungus Clonostachys rosea, due its potential to be used in agriculture. The fungus can be found in different regions, colonizing all parts of many vegetable species, and also has the ability of remaining endophytic in some plant species. Regarding biological control, C. rosea is proven to be effective against different pathogens in a wide host range. Moreover, there are reports of the antagonist working as a growth promoter, inducing resistance in some plant species and being an entomopathogenic fungus. Due to the efficiency and versatility of $C$. rosea, we attempt in this review to provide a general overview on the potential usage of the antagonist in the context of biological control.
\end{abstract}

Keywords: Biological control, antagonists, endophytic fungi, Bionectria ochroleuca

\section{INTRODUÇÃO}

O uso de pesticidas na agricultura vem sofrendo restrições, principalmente em vista dos resíduos em alimentos, seleção de populações de patógenos resistentes, contaminação ambiental e dos riscos à saúde humana. Assim, medidas alternativas e eficientes no controle de doenças de plantas são necessárias, como o controle biológico. Embora a quantidade de produtos comerciais à base de microrganismos antagonistas ainda seja pequena, representando $1 \%$ do mercado mundial de pesticidas, enquanto os fungicidas representam aproximadamente $15 \%$ do mercado, o uso de agentes de biocontrole vem crescendo, principalmente no contexto do manejo integrado de doenças (Fravel, 2005). Com o uso de antagonistas pode-se reduzir a necessidade da aplicação de fungicidas, combinando o controle químico com a aplicação destes agentes e, consequentemente, reduzindo a possibilidade de seleção de isolados de patógenos resistentes a fungicidas, o que torna o controle mais estável e sustentável (Fravel, 2005; Shtienberg e Elad, 1997). Além disso, ocorre redução de resíduos químicos nos alimentos, menor exposição dos aplicadores a agrotóxi- cos e menor contaminação do ambiente (Pertot et al., 2008). Agentes de biocontrole, além de reduzir o efeito dos patógenos nas plantas, podem promover outros efeitos benéficos, que incluem a melhoria das condições fisiológicas, redução de estresses abióticos, melhoria da eficiência fotossintética, aumento da absorção de água e assimilação de nutrientes, o que torna as plantas mais saudáveis e produtivas (Schroers et al., 1999).

Dentre os microrganismos com potencial de uso na agricultura, vem-se dando ênfase ao fungo Clonostachys rosea (Link Fries). O potencial de uso agrícola de $C$. rosea deve-se ao fato de esse microrganismo controlar diversos patógenos fúngicos (Cota et al., 2008b; Krauss e Soberanis, 2001; Lahoz et al., 2004; Lubeck et al., 2002; Morandi et al., 2008; Sutton et al., 1997; Xue, 2003), ser de fácil produção em condições controladas (Saraiva, 2009), poder parasitar insetos (Toledo et al., 2006; Vega et al., 2008) e cistos de nematóides dos gêneros Heterodera e Globodera (Sutton et al., 1997), além de ser endofítico e promover crescimento de plantas (Lubeck et al., 2002; Morandi et al., 2001; Sutton et al., 2008; 2002). 
Nas últimas décadas, grupos de pesquisa de diferentes países se dedicaram ao estudo desse antagonista, visando compreender aspectos ecológicos, mecanismos envolvidos no controle biológico, interações de C. rosea com as espécies vegetais, visando a promoção de crescimento e indução de resistência, e a compatibilidade de uso do antagonista associado a outros métodos de controle. Diversos resultados promissores para o uso de C. rosea na agricultura foram obtidos, entretanto ainda existem gargalos para sua utilização em massa.

Nesta revisão, objetiva-se sintetizar os avanços obtidos na pesquisa para o uso de C. rosea na agricultura, com a definição dos requerimentos autoecológicos, aspectos para a produção massal e vida de prateleira, eficiência do antagonista em condições de casa de vegetação e campo, efeitos benéficos sobre as plantas como indução de resistência e promoção de crescimento, além de mencionar perspectivas futuras e dificuldades ainda existentes para maior adoção do antagonista.

\section{CONTROLE BIOLÓGICO}

O controle de doenças de plantas, por muitas décadas, objetivou eliminar patógenos através do uso contínuo de produtos químicos. Isso resultou no uso indiscriminado de pesticidas como única medida de controle de doenças de plantas. Hoje, a busca por métodos mais sustentáveis no controle de doenças vem aumentando e ganhando importantes nichos de mercado, pois o uso excessivo de agrotóxicos, sem um plano de manejo, não é a melhor opção para uma agricultura sustentável. Na busca por métodos sustentáveis, que envolve o manejo adequado dos recursos naturais e o cultivo menos dependente do uso de agrotóxicos, o controle biológico, "redução da densidade de inóculo ou das atividades determinantes da doença por um ou mais organismos, realizado naturalmente ou através da manipulação do ambiente, hospedeiro ou antagonista, ou pela introdução em massa de um ou mais antagonistas" (Baker e Cook, 1974), é um dos métodos mais pesquisados.

Apesar de antigo, com mais de 100 anos (Baker e Cook, 1974), o uso de controle biológico aplicado com a introdução em massa de microrganismos é recente e a quantidade de produtos comerciais disponíveis no mercado ainda é pequena, representando menos que $1 \%$ dos produtos usados na agricultura. Embora $\mathrm{O}$ número de produtos e a porcentagem de comercialização sejam baixos, a importância de agentes de biocontrole vem aumentando, e espera-se um aumento anual de, aproximadamente, $10 \%$ na taxa de comercialização de produtos de origem biológica, incluindo agentes de biocontrole, semioquímicos e feromônios (Bailey et al., 2010). Com maior número destes produtos disponíveis no mercado, espera-se menor dependência de fungicidas. Em alguns casos, o uso de um agente de biocontrole pode ser o melhor método de manejo de doenças (Shtienberg e Elad, 1997; Elad e Stewart, 2004; Fravel, 2005).

Entretanto, a adoção do controle biológico requer maiores conhecimentos. Por exemplo, o entendimento da ecologia do patógeno e do antagonista, domínio das técnicas de cultivos em grande escala, com manutenção da eficiência das atividades do antagonista, domínio das técnicas de aplicação, desenvolvimento de equipamentos específicos, aumento da vida de prateleira e integração com outros métodos. Desta forma, estudar os efeitos climáticos que influenciam o antagonista, a interação antagonista vs patógeno vs planta e a produção em larga escala é crucial para o sucesso do método. Há muitos casos de sucesso com uso de agentes de biocontrole na agricultura e muitos agentes microbianos registrados em diferentes países (Montesinos, 2003). Na busca 
por antagonistas que sejam eficientes, grande ênfase vem sendo dada ao fungo Clonostachys rosea. Esta revisão aborda as principais características e a versatilidade desse fungo e seu uso no controle biológico.

\section{Clonostachys rosea: TAXONOMIA, ECOLOGIA E USO}

Clonostachys rosea (Link Fries) [forma perfeita = Bionectria ochroleuca], anteriormente classificado como Gliocadium roseum (Schroers et al., 1999), produz conídios unicelulares em conidióforos de dois tipos, penicilado e verticilado. As colônias do fungo em meio de cultura são geralmente brancas, alaranjadas ou salmão, coloração próximo ao rosa e que dá o nome à espécie. A fase sexuada, B. ochroleuca, é incluída na classe Ascomycetes, ordem Hypocreales.

Clonostachys rosea é um organismo cosmopolita, presente naturalmente no solo, restos culturais e plantas daninhas, sendo encontrado em regiões temperadas, tropicais e desérticas (Nobre et al., 2005; Sutton et al., 1997). Já foi relatado em culturas, campo, matas, florestas, água-doce e solos litorâneos, particularmente em locais com $\mathrm{pH}$ variando entre neutro e alcalino (Sutton et al., 1997). O fungo é conhecido por colonizar raízes, hastes, vagens, folhas e sementes de diversas espécies de plantas e ter a capacidade de permanecer endofiticamente. Por exemplo, obteve-se o antagonista a partir de hastes, vagens, folhas e sementes de soja (Schiller y Sinclair, 1984), raízes de trevo vermelho, folhas de morango e framboesa (Sutton et al., 1997). C. rosea foi endofitico em raízes de cevada (Lubeck et al., 2002), tecidos verdes de roseira (Morandi et al., 2001) e tomateiro (Sutton et al., 2002; 1997). Além de colonizar plantas, C. rosea coloniza cistos de Heterodera ssp., Globodera ssp. e outras espécies de nematóides (Jones et al., 1986; Kennerley et al., 1987) e escleródios de fungos de diversas espécies (Stewart y Harrison, 1989; Keinath et al.,1991;
Sutton y Peng, 1993). O fungo é eficiente em colonizar solos e plantas em ampla faixa de temperatura, variando de 18 a $30^{\circ} \mathrm{C}$. Entretanto, para o morango, a maior eficiência ocorreu a $24^{\circ} \mathrm{C}$ (Cota et al., 2008b). Bionectria ochroleuca, a fase sexuada, é encontrada principalmente em ramos mortos de árvores, em plantas herbáceas e tecidos carnosos de plantas e fungos (Sutton et al., 1997).

Para aumentar a eficiência do uso de antagonistas, é de grande importância compreender as características e os mecanismos de ação dos agentes envolvidos no processo (Elad e Stewart, 2004; Fravel, 2005). O agente de controle ideal seria aquele com diferentes modos de antagonismo. Clonostachys rosea apresenta diferentes mecanismos, como competição por nutrientes e substratos, micoparasitismo, produção de enzimas eficientes no controle de patógenos, entomopatogenicidade e indução de resistência o que permite sua utilização frente a diferentes patógenos e aumenta sua possibilidade de sucesso.

Os estudos para uso do antagonista concentram-se no que diz respeito ao controle de fungos fitopatogênicos, principalmente por competição por nutrientes e espaço ou por micoparasitismo (Yu e Sutton, 1997). Como exemplo, tem-se o controle de Aphanomyces euteiches, Alternaria alternata, Alternaria dauci, Alternaria radiciana, Botrytis cinerea, Erysiphe orontii, Fusarium culmorum, Fusarium oxysporum f. sp. pisi, Fusarium solani f. sp. pisi, Moniliophthora roreri, Moniliophthora perniciosa, Mycosphaerella pinodes, Phytophthora palmivora, Pythium spp., Rhizoctonia solani e Sclerotinia sclerotiorum (Sutton et al., 1993,1997; Tatagiba et al.,1998; Jensen et al., 2000; Krauss e Soberanis, 2001; Morandi et al., 2001; Lubeck et al., 2002; Sutton et al., 2002; Jensen et al., 2002; Morandi et al., 2003; Xue, 2003; Lahoz et al., 2004; Nobre et al., 2005; Morandi et al., 2006; Cota et al., $2008 \mathrm{ab}$; Morandi et al., 2008; Roberti et al., 2008; Mamarabadi et 
al., 2009; Correa et al., 2010; Rodríguez et al., 2011).

Relacionado ao micoparasitismo, hifas de C. rosea podem parasitar conídios, escleródios e hifas de B. cinerea (Sutton et al., 1997). Hifas do antagonista podem estrangular hifas de B. cinerea (Morandi et al., 2001) e, após penetração direta, promover a ruptura e degradação das células do patógeno (Li et al., 2002). Também se constatou o micoparasitismo em Moniliophthora roreri, Moniliophthora perniciosa e Phytophthora palmivora (Krauss e Soberanis, 2001). Em estudo "in vitro", observou-se que $C$. rosea parasitou Alternaria alternata, Aphanomyces euteiches, Fusarium oxysporum f. sp. pisi, F. solani f. sp. pisi, Mycosphaerella pinodes, Pythium spp., Rhizoctonia solani, e Sclerotinia sclerotiorum, fungos responsáveis por provocar o complexo de podridão radicular da ervilha, doença de difícil controle e o principal fator limitante na produção da cultura no Canadá (Xue, 2003). Em condições controladas, a aplicação do antagonista em sementes de ervilha aumentou a germinação e emergência em 44 e $22 \%$, respectivamente. Neste trabalho, observou-se redução da severidade da doença em $76 \%$, redução superior à com aplicação de thiram, de $65 \%$. Quando se aplicaram os tratamentos no campo, ambos foram eficientes no controle da doença em relação à testemunha e não diferiram entre si (Xue, 2003).

Sob condição controlada, C. rosea também foi eficiente no controle de Sclerotinia sclerotiorum em plantas de alface e soja (Rodriguez et al., 2011). Os autores concluíram que C. rosea atuava principalmente por antibiose, produzindo compostos antifúngicos associados ao metabolismo secundário, e por micoparasitismo (Rodriguez et al., 2011).

Além do micoparasitismo, a competição por espaço e nutrientes é considerada por alguns autores como o principal mecanismo de antagonismo de C. rosea (Sutton e Peng, 1993; Morandi et al., 2001, 2003). Por exemplo, o antagonista colonizou as sementes de ervilha e se estabeleceu nas raízes, impedindo a infecção por patógenos (Xue, 2003). Segundo Sutton et al. (1997) o antagonista compete por nutrientes e nicho com $B$. cinerea, o que inibe a germinação e estabelecimento do patógeno. (Sutton et al., 1997). Este mecanismo de antagonismo foi comprovado contra B. cinerea em begônia, tomate, gerânio, framboesa e morango (Sutton et al., 1997). O isolado canadense PG-88-710 reduziu em mais de $99 \%$ a esporulação de $B$. cinerea em folhas de roseira, mesmo quando aplicado 24 h após a inoculação do patógeno (Morandi et al., 2001). Este fato que comprova a habilidade competitiva de $C$. rosea, pois o fungo pode competir com o patógeno mesmo quando este já está presente (Morandi et al., 2001). O isolado PG-88-710 também atuou por competição e reduziu a esporulação de $B$. cinerea em morangueiro, eucalipto e roseira (Valdebenito-Sanhuenza et al., 1997; Von Stowasser e Ferreira, 1997; Morandi et al., 2001; Morandi et al., 2003).

A maioria dos estudos com C. rosea tem sido baseada no controle de fungos. Um dos patógenos mais estudados é $B$. cinerea. $\mathrm{O}$ antagonismo deste fungo por $C$. rosea em vários hospedeiros ocorre em vista de o antagonista se estabelecer nos tecidos de plantas e competir com o patógeno na ausência de molhamento foliar, em períodos prolongados de molhamento foliar, independente do estádio de desenvolvimento dos tecidos e possuir requerimentos de temperaturas próximos aos do patógeno (Morandi et al., 2000; Morandi et al., 2003; Morandi et al., 2006; Morandi et al., 2008; Cota et al., 2008b).

O sucesso obtido no controle biológico de B. cinerea por $C$. rosea estimulou a busca por novos isolados que fossem adaptados às condições regionais. No Brasil, estudos de prospecção resultaram na obtenção de quatro isolados do antagonista (NCR19/F, NCR60/F, NCR61/F, NCR62/F) (Nobre et al., 2005). Estes isolados 
foram eficientes em competir e reduzir a esporulação de $B$. cinerea em folhas de morangueiro, roseira, eucalipto e tomateiro (Nobre et al., 2005). A vantagem competitiva desses isolados sobre $B$. cinerea foi garantida pelo fato destes colonizarem folhas de morangueiro em curtos ou longos períodos de molhamento foliar (Cota et al., 2008b). Em experimentos de campo, realizados no município brasileiro de Viçosa-MG, os quatro isolados controlaram eficientemente $B$. cinerea em folhas, flores e frutos de morangueiro. A aplicação semanal da mistura dos quatro isolados foi tão eficiente quanto à pulverização de fungicidas em duas aplicações semanais, e resultou em maior nível de controle e incrementos superiores a $100 \%$ da produção (Cota et al., 2008a). Em temperaturas inferiores a $10^{\circ} \mathrm{C}, \mathrm{C}$. rosea foi pouco eficiente em colonizar e competir com B. cinerea (Cota et al., 2008 b), provavelmente pela redução da habilidade competitiva do antagonista (Köhl et al., 1999), mas em temperatura média em torno de $16^{\circ} \mathrm{C}$, não houve limitação da atuação do antagonista. Alta incidência de luz solar associada à baixa umidade relativa reduziu a germinação de C. rosea, porém mesmo quando aplicado em condições adversas em casa de vegetação (alta radiação solar, baixa umidade) o antagonista estabeleceu-se e controlou B. cinerea (Morandi et al., 2008).

Clonostachys rosea também produz compostos antifúngicos e enzimas que degradam a parede de fungos, mas o papel dessas substâncias no parasitismo ainda não foi completamente elucidado (Sutton et al., 1997). O antagonista produz, principalmente, proteases e quitinases, enzimas importantes no processo de infecção de nematóides e de insetos (Li et al., 2006; Mamarabadi et al., 2009; Lianming et al., 2011). É provável que o antagonista produza outros compostos, que podem degradar paredes de oomicetos, como Pythium ultimum (Mamarabadi et al., 2009). Compostos do metabolismo secundário produzidos por C. rosea são tam- bém usados no parasitismo de fungos. Em pareamento de culturas do isolado BAFC3874 do antagonista e Sclerotinia sclerotiorum, observouse que $C$. rosea atua principalmente produzindo compostos antifúngicos associados ao metabolismo secundário (Rodriguez et al., 2011).

O potencial de uso do antagonista não se restringe ao controle de doenças fúngicas. Clonostachys rosea também é eficiente no controle biológico de nematóides, principalmente de espécies de Meloidogyne. O fungo pode parasitar ovos e pode produzir dois tipos de enzimas, protease e quitinase, que são chaves para o antagonismo (Khan et al., 2006). Além disso, o fungo pode penetrar diretamente na cutícula do nematóide após a formação do apressório. Já se observou cistos de Heterodera spp, e Globodera spp. parasitados por C. rosea (Sutton et al., 1997) e outros nematóides tanto de plantas como de animais (Zhang et al., 2008; Khan et al., 2006; Zou et al., 2010 ab; Baloyi et al., 2011). Por meio de microscopia de fluorescência, um isolado transformado de C. rosea, expressando a green fluorescent protein (GFP), foi antagonista ao nematóide Panagrellus redivivus (Zhang et al., 2008). Os conídios do antagonista aderiram-se à cutícula do nematóide e, após iniciar a germinação, os tubos germinativos penetraram no nematóide, levando-o à imobilização e, posteriormente, à morte. Adicionalmente, no corpo do nematóide houve produção intensa de micélio e esporos, o que pode gerar novos ciclos, inclusive em outros nematóides.

Clonostachys rosea também é considerado entomopatogênico. $\mathrm{O}$ antagonista parasitou a broca do cafeeiro quando aplicado em insetos adultos. Estes morreram, havendo formação de micélio e de esporos do antagonista, sendo o ciclo desde a inoculação até esporulação de 16 dias (Vega et al., 2008). Na Argentina, encontrou-se $C$. rosea parasitando Oncometopia tucumana (Hemiptera) e Sonesimia grossa (Cicadellidae), vetores importantes de Xylella 
fastidiosa, agente causal da clorose variegada dos citros (Toledo et al., 2006). Os autores isolaram o fungo, aplicaram-no em insetos de diferentes idades e, após 14 dias, detectaram-se mortalidade de $82,5 \%$ e $45,5 \%$ em 0 . tucumana e $S$. grossa, respectivamente.

Outra característica importante de C. rosea é sua capacidade de promover crescimento de diferentes espécies de plantas. A aplicação do isolado canadense $\mathrm{Pg}$ 88-710 de C. rosea aumentou o crescimento e a produção de roseiras, gerânios, pepinos (Sutton et al., 2008) e tomateiros (Sabine et al., 2006). Os autores analisaram os nutrientes em folhas e detectaram aumento do conteúdo de fósforo e redução de nitrogênio após a aplicação do antagonista. A aplicação do antagonista também aumentou a matéria de tomateiros, avaliadas 30 dias após o semeio (Macedo, 2011). Aparentemente, a interação antagonista $x$ espécies de plantas ou isolados do antagonista $x$ espécies de plantas é específica, e há relatos de insucesso. Por exemplo, o antagonista reduziu a incidência da podridão das raízes, causada por Pythium aphanidermatum, mas não promoveu o crescimento de plantas de alface cultivadas hidroponicamente (Correa et al., 2010).

Um aspecto importante na adoção de controle biológico é sua compatibilidade de uso com outros métodos. A integração de $C$. rosea, com remoção dos restos culturais e controle químico no manejo do mofo cinzento do morangueiro em condições de campo, foi o tratamento mais eficiente e a aplicação do agente de controle biológico foi o elemento chave no manejo (Cota et al., 2009). Estabelecer e explorar mais rápido o substrato que o patógeno, garante vantagem competitiva o que leva a redução do patógeno (Cota et al., 2009).

Recentemente, observou-se que o antagonista também induziu resistência a patógenos em algumas espécies de plantas. Após tratar raí- zes de plantas de tabaco com C. rosea, analisou-se o extrato foliar das plantas quanto aos níveis de $\beta$-1,3-glucanases, $\beta$-1,4-glicosidases, quitinases, $\mathrm{N}$-acetil-b-glucosaminidase e peroxidases (Lahoz et al., 2004). As atividades de $\beta$-1,3-glucanases, $\beta$-1,4-glicosidases e quitinases aumentaram nas plantas tratadas com o antagonista. Os autores também concluíram que a severidade do oídio foi menor em plantas tratadas e inoculadas com Erysiphe orontii nas folhas, que em plantas não tratadas. Esta foi a primeira constatação de C. rosea como indutor de resistência de plantas. Também observouse indução de resistência em trigo à Fusarium culmorum. Aplicou-se o antagonista nas sementes e inoculou-se o patógeno. Analisaramse proteínas (PR4), peroxidases e quitinases. Com aplicação de C. rosea, as plantas produziram os compostos de defesa mais rapidamente, induzindo resistência em trigo (Roberti et al., 2008). A produção rápida de compostos de defesa é de grande importância, pois em alguns casos as plantas conseguem reconhecer o patógeno e iniciar sua defesa. Porém, em certas ocasiões o ataque é muito rápido e, quando a planta produz os compostos de defesa, a infecção já está adiantada, e o patógeno não é inativado. A aplicação de C. rosea promoveu também, maior e mais vigoroso crescimento das raízes de trigo, provavelmente, porque $C$. rosea reduz a atividade de fungos prejudiciais e favorece a absorção de nutrientes (Roberti et al., 2008).

Uma vantagem competitiva de C. rosea é sua capacidade de permanecer endofítico em tecidos verdes (Sutton et al., 1997; Morandi et al., 2000; Morandi et al., 2001). Entretanto, até então, as avaliações para comprovar a capacidade endofítica de $C$. rosea eram indiretas, geralmente por meio de desinfestação superficial e deposição em meio PCA (Sutton et al., 2002). Recentemente, realizou-se um estudo para entender a interação do antagonista com B. cinerea no tomateiro, usando microscopia confocal. 
Observou-se, através da secção de fragmentos, que o antagonista colonizou os espaços intercelulares de haste de tomateiro e foi capaz de se multiplicar internamente nos tecidos (dados não publicados). Assim, além de se estabelecer nos tecidos e os colonizar, $C$. rosea se reproduz nos tecidos da planta hospedeira, o que explica, em parte, porque o antagonista permanece na planta por longos períodos. A capacidade endofítica de C. rosea possibilita ao antagonista já estar presente quando da chegada do patógeno e reduzir sua exposição a condições adversas do meio ambiente. Geralmente, fungos endofíticos proporcionam às plantas hospedeiras maior proteção a patógenos e insetos e melhoram a absorção de nutrientes (Vega et al., 2008; Elmi et al., 2000; Wicklow et al., 2005).

Em síntese, C. rosea é um organismo cosmopolita, antagonista por meio de vários mecanismos a diversos fungos patogênicos, nematóides e insetos e que pode promover o crescimento e a indução de resistência em plantas. Em vista destas características, acredita-se que C. rosea seja competitivo e adaptável, podendo ser usado em diferentes ambientes. Nos Estados Unidos e no Canadá já existe um produto registrado a base de C. rosea, chamado Endofine. No Brasil, há um produto à base do antagonista, Clonosnat, que ainda não é registrado no Ministério da Agricultura.

\section{PRODUÇÃO EM LARGA ESCALA}

Há vários aspectos cruciais para viabilizar o biocontrole com microrganismos. Dentre esses, é importante desenvolver protocolo de produção do inóculo em grande escala, o que envolve obter uma formulação estável do microrganismo e de um substrato apropriado à produção, bem como determinar as condições de cultivo e de estocagem (Elad e Stewart, 2004). Uma das mais importantes é a de produção, incluindo a seleção do meio, o tipo de processo utilizado na produção (fermentação em estado sólido, semi-sólido e líquido) e as condições ótimas para o cultivo (temperatura, umidade, atividade de água, pH, aeração e agitação, entre outras) (Viccini, 2004).

O objetivo da produção massal é obter a maior quantidade de propágulos eficazes no menor período de tempo. Um possível limitante do processo é o custo, em vista do valor de substrato ou do processo de produção em grande escala (Fravel, 2005). Assim, a busca por substratos baratos, eficientes na produção massal e de fácil utilização é crucial no desenvolvimento de produtos à base de agentes de biocontrole. Podem-se produzir propágulos de um antagonista por meio de fermentação líquida, difásica ou sólida. A fermentação sólida consiste no uso de substratos como grãos de cereais e resíduos agrícolas, com pequenas quantidades de água no substrato e ausência de água livre (Pandey, 2003). A produção de propágulos em estado sólido é vantajosa, em vista do baixo custo, praticidade e fácil execução. Ademais, esporos produzidos por meio de fermentação sólida são mais robustos e com maior longevidade que os produzidos em fermentação líquida (Jensen et al., 2002).

No processo de fermentação, o meio de cultivo deve satisfazer a critérios como a disponibilidade de nutrientes, fácil preparo e esterilização e baixo custo. Além da disponibilidade de nutrientes, os níveis de umidade e a superfície dos substratos sólidos podem influenciar tanto a quantidade quanto a qualidade dos esporos produzidos. Há vários substratos orgânicos, como cereais, para a produção massal de antagonistas (Viccini, 2004; Fravel, 2005).

Comumente, produzem-se os esporos de $C$. rosea com a metodologia descrita por Sutton y Yu (1994): C. rosea cresce em grãos de trigo durante 35 dias em média, a $20-23^{\circ} \mathrm{C}$. Vemse testando outros substratos e metodologias de produção nos últimos anos. Viccini (2004), 
usando grãos de trigo e arroz para produção de esporos de $C$. rosea, observou que a produção de esporos, em 40 dias de fermentação, obtida usando arroz como substrato $\left(1,0 \times 10^{9}\right.$ esporos/g substrato) foi uma ordem de magnitude maior que aquela obtida com grãos de trigo $\left(1,5 \times 10^{8}\right.$ esporos/g substrato). Sutton et al. (1997) obtiveram maior produção de esporos, usando grãos de trigo como substrato (1 a $5 \times 10^{9}$ esporos/g obtido em 35 dias de fermentação), enquanto Krauss e Soberanis (2001) usaram arroz como substrato eficaz para produção massal de propágulos de C. rosea.

Avaliar substratos alternativos e ao mesmo tempo eficientes para multiplicar C. rosea é de grande importância. Morandi (1997) avaliaram grãos de trigo, arroz com casca, arroz integral e folhas de roseira, e observaram esporulação abundante nas folhas de roseiras, após 15 dias (3,9 × $10^{8}$ conídios/g); nos grãos, esporulação abundante ocorreu somente após 40 dias (5,7 x $10^{8} ; 6,1 \times 10^{8} ; 6,4 \times 10^{8}$ conídios/g de trigo, arroz com casca e arroz integral, respectivamente). Em outro trabalho, adotou-se mistura de turfa, farelo de trigo e água (15:26:59) na produção de conídios do isolado IK726 de C. rosea (Jensen et al., 2002). No Brasil, na região de Bento Gonçalves, RS, C. rosea é produzido em escala semi-comercial pela Embrapa Uva e Vinho (Valdebenito-Sanhueza et al., 1997).

No processo de formulação, o desempenho do agente de biocontrole pode ser afetado, podendo haver aumento ou redução de eficiência do controle, além de afetar a vida útil e a segurança do produto (Fravel, 2005). A formulação de antagonistas para uso comercial geralmente envolve a mistura de propágulos viáveis com materiais inertes, como caolim, e fonte de nutrientes, havendo produção de pós molháveis ou grânulos. Os materiais inertes podem ajudar a estabilizar a formulação, podendo aumentar o período de estocagem e facilitar o uso na aplicação. A natureza do antagonista, o patógeno alvo, o local de ação e da aplicação são os principais fatores que determinam o tipo de formulação (Sabaratnam e Traquair, 2002).

O período de vida de prateleira é crucial para o sucesso comercial de uma formulação à base de microrganismos. Recomenda-se prazo de validade mínimo de 1 ano, com o mínimo de perdas na viabilidade, de preferência sem refrigeração (Sarembaud e Feinberg, 2007). A preservação de produtos biológicos é fortemente influenciada por fatores externos, principalmente, oxigênio, dióxido de carbono, etileno, umidade relativa do ar, pressão ou estresse mecânico, luz e temperatura (Sarembaud e Feinberg, 2007).

A umidade e temperatura são fatores importantes no armazenamento do agente de biocontrole (Wraight et al., 2001). A secagem rápida de conídios pode ser estratégia eficiente para manter a viabilidade dos propágulos por um período maior. Como relatado por alguns autores, a viabilidade de C. rosea foi maior quando os conídios foram submetidos à secagem rápida, após produção massal em 20 dias em mistura de turfa, farelo de trigo e água (Jensen et al., 2002).

A eficiência no armazenamento de uma formulação também depende da embalagem de armazenamento. Por exemplo, uma formulação com baixa umidade ou armazenada a vácuo, requer embalagens impermeáveis, sendo a de folhas laminadas de polietileno-alumínio as mais adequadas. Em contraposição, para formulações em que o oxigênio é necessário para sobrevivência do antagonista, embalagens de polietileno são eficientes. Estas permitem as trocas gasosas e formam barreira contra perda e ganho de umidade. Sacolas plásticas, sacos de alumínio, potes de polipropileno são embalagens já utilizadas no armazenamento de $C$. rosea. Pode-se usar dessecantes como sílica gel nas formulações, o que melhora a capacidade de armazenamento em temperatura ambiente (Jensen et al., 2002). 


\section{CONCLUSÃO}

A busca por organismos eficientes no controle biológico de doenças e a demonstração da eficiência dos mesmos têm sido o foco de pesquisas nas últimas décadas. Clonostachys rosea é um destes agentes com comprovada eficiência no controle de vários patógenos. Este fungo é amplamente distribuído, atua por diversos mecanismos de antagonismo, pode crescer endofiticamente em espécies vegetais, tem diversas vantagens ecológicas e esporula abundantemente em alguns substratos. Estas características favorecem o uso do antagonista como agente de biocontrole. Pesquisas com epidemiologia, toxicologia e efeitos ao ambiente em diferentes patossistemas são necessárias para aumentar a disponibilidade de produtos à base de C. rosea. A interação entre a planta, o patógeno e o antagonista deve ser bem entendida, assim como formulações estáveis e eficientes devem ser desenvolvidas para que o uso do antagonista seja potencializado. O produto formulado com $C$. rosea deve fazer parte de um manejo integrado de doenças, para que o mesmo tenha aceitação do agricultores, gerando condição ideal de uso do produto e contribuindo para o estabelecimento de uma agricultura economicamente viável e ecologicamente sustentável.

\section{BIBLIOGRAFÍA}

BAILEY, K.L., BOYETCHKO, S.M. y LANGLE, T. 2010. Social and economic drivers shaping the future of biological control: A Canadian perspective on the factors affecting the development and use of microbial biopesticides. Biological Control. 52:221 - 229.

BALOYI, M.A., LAING, M.D. y YOBO, K.S. 2011. Use of mixed cultures of biocontrol agents to control sheep nematodes M.A. Veterinary Parasitology. 184:2 - 4.
BAKER, K.F. y COOK, R.J. 1974. Biological control of pathogens. San Francisco: Freeman and Company. 433 p.

COTA, L.V., MAFFIA, L.A., MIZUBUTI, E.S.G. y MACEDO, P.E.F. 2009. Biological control by Clonostachys rosea as a key component in the integrated management of strawberry gray mold. Biological Control. 50:222 - 230.

COTA, L.V., MAFFIA, L.A., MIZUBUTI, E.S.G. 2008a. Brazilian isolates of Clonostachys rosea: colonization under different temperature and moisture conditions and temporal dynamics on strawberry leaves. Letters in Applied Microbiology. 46:312 - 317.

COTA, L.V., MAFFIA, L.A., MIZUBUTI, E.S.G., MACEDO, P.E.F. y ANTUNES, R.F. 2008b. Biological control of strawberry gray mold by $\mathrm{Clo}-$ nostachys rosea under field conditions. Biological Control. 46:515 - 522.

CORREA, E.B., BETTIOL, W. y MORANDI, M.A.B. 2010. Biological control of Pythium aphanidermatum root rot and growth promotion of hydroponic lettuce by Clonostachys rosea. Tropical Plant Pathology.35:248 - 252.

ELAD, Y. y STEWART, A. 2004. Microbial Control of Botrytis. In: Elad Y, Williamson B, Tudzynski P, Delen N. Botrytis: Biology, Pathology and Control. Kluwer Academic Publishers. $223-236$ p.

ELMI, A.A., WEST, C.P., ROBBINS, R.T. y KIRKPATRICK, T.L. 2000. Endophyte effects on reproduction of a root-knot nematode (Meloidogyne marylandi) and osmotic adjustment in tall fescue. Grass and Forage Science. 55:166 $-172$.

FRAVEL, D.R. 2005. Commercialization and implementation of biocontrol. Annual Review of Phytopathology. 43:337 - 359. 
JENSEN, B., KNUDSEN, I.M.B. y JENSEN, D.F. 2002. Survival of conidia of Clonostachys rosea on stored barley seeds and their biocontrol efficacy against seed-borne Bipolaris sorokiniana. Biocontrol Science and Technological. 12:427 - 441.

JENSEN, B., KNUDSEN, I.M.B. y JENSEN, D.F. 2000. Biological seed treatment of cereals with fresh and long-term stored formulations of Clonostachys rosea: Biocontrol efficacy against Fusarium culmorum. European Journal of Plant Pathology. 106: 233 - 242.

JONES, G.M., RODRIGUEZ-KABANA, R. y JATALA P. 1986. Fungi associated with cysts of potato cyst nematodes in Peru. Nematropica. 16:21 - 31 .

KENNERLEY, C.M., JEGER, M.J., ZUBERER, D.A. y JONES, R.W. 1987. Populations of fungi associated with sclerotia of Phymatotrichum omnivorum in Houston black clay. Transactions of the British Mycological Society. 89:437 - 445.

KEINATH, A.P., FRAVEL, D.R. y PAPAVIZAS, G.C. 1991. Potential of Gliocladium roseum for biocontrol of Verticillium dahliae. Phytopathology. 81:644 - 648.

KHAN, A., WILLIAMS, K.L. y NEVALAINEN, H.K.M. 2006. Infection of plant-parasitic nematodes by Clonostachys rosea and Monacrosporium lysipagum. Biological Control. 51:659 - 678.

KÖHL, J., LOMABERS-VAN DER PLAS CH. y MOLHOEK, W.M.L., KESSEL, G.J.T. 1999. Competitive ability of the antagonists Ulocladium atrum and Gliocladium roseum at temperatures favourable for Botrytis spp. development. Biocontrol. 44:329 - 46.

KÖHL, J. y FOKKEMA, N,J. 1998. Biological control of Botrytis cinerea by supression of sporulation. In: The 1998 Brighton Conference Pests \& Diseases, Proceedings: 681 - 686.
KRAUSS, U. y SOBERANIS, W. 2001. Biocontrol of cocoa pod diseases with mycoparasite mixtures. Biological Control. 22:149 -158.

LAHOZ, E., CONTILLO, R. y PORRONE, F. 2004. Induction of systemic resistance to Erysiphe orontii cast in tobacco by application on roots of an isolate of Gliocladium roseum Bainier. Journal of Phytopathology.152:465 - 470.

LI, G.Q., HUANG, H.C., KOKKO, E.G. y ACHARYA, S.N. 2002. Ultrastructural study of mycoparasitism of Gliocladium roseum on Botytis cinerea. Botanical Bulletin of Academia Sinica. 43:211 - 218.

LI, J., YANG, J., HUANG, X. y ZHANG, K.Q. 2006. Purification and characterization of an extracellular serine protease from Clonostachys rosea and its potential as a pathogenic factor. Process Biochemistry. 41:925 - 929.

LIANMING, L., JINKUI, Y., JUAN, L., M.O, Y., LI, L., ZHAO, X. y ZHANG, K.Q. 2011. Cloning and homology modeling of a serine protease gene $(\mathrm{PrC})$ from the nematophagous fungus Clonostachys rosea. Annals of Microbiology . 61:511 - 516.

LUBECK, M., KNUDSEN, I.M.B., JENSEN, B., THRANE, U., JANVIER, C. y JENSEN, D.F. 2002. GUS and GFP transformation of the biocontrol strain Clonostachys rosea IK726 and the use of these marker genes in ecological studies. Mycological Research. 106:815 - 826.

MACEDO, P.E.F. 2011. Promoção de crescimento de plantas mediada por Clonostachys rosea. Dissertação de Mestrado, Universidade Federal de Viçosa. Viçosa, MG, Brasil.

MAMARABADI, M., JENSEN, D.F. y LUBECK, M. 2009. An N-acetyl-b-D-glucosaminidase gene, cr-nag1, from the biocontrol agent Clonostachys rosea is up-regulated in antagonistic 
interactions with Fusarium culmorum. Mycological Research. 113:33 - 43.

MONTESINOS, E. 2003. Development, registration and commercialization of microbial pesticides for plant protection. International Microbiology. 6:245 - 252.

MORANDI, M.A.B., MATTOS, L.P.V., SANTOS, E.R. y BONUGLI, R.C. 2008. Influence of application time on the establishment, survival, and ability of Clonostachys rosea to suppress Botrytis cinerea sporulation on rose debris. Crop Protection. 27:77 - 83.

MORANDI, M.A.B., MAFFIA, L.A., MIZUBUTI, E.S.G., ALFENAS, A.C., BARBOSA, J.G. y CRUZ, C.D. 2006. Relationships of microclimatic variables to colonization of rose debris by Botrytis cinerea and the biocontrol agent Clonostachys rosea. Biocontrol Science and Technology. 16:619 - 630 .

MORANDI, M.A.B., MAFFIA, L.A., MIZUBUTI, E.S.G., ALFENAS, A.C. y BARBOSA, J.G. 2003. Suppression of Botrytis cinerea sporulation by Clonostachys rosea on rose debris: a valuable component in Botrytis blight managenent in commercial greenhouses. Biological Control. $26: 311$ - 317 .

MORANDI, M.A.B., MAFFIA, L.A. y SUTTON, J.C. 2001. Development of Clonostachys rosea and interactions with Botrytis cinerea in rose leaves and residues. Phytoparasitica. 29:1 - 11.

MORANDI, M.A.B., SUTTON, J.C. Y MAFFIA L.A. 2000. Effects of host and microbial factors on development of Clonostachys rosea and control of Botrytis cinerea in rose. European Journal of Plant Pathology. 106:439 - 448.

MORANDI, M.A.B. 1997. Gliocladium roseum como agente de biocontrole de Botrytis cinerea em roseiras cultivadas em casa de vegetação.
Dissertação de Mestrado, Universidade Federal de Viçosa. Viçosa MG, Brasil.

NOBRE, S.A.M., MAFFIA, L.A., MIZUBUTI, E.S.G., COTA, L.V. y DIAS, A.P.S. 2005. Selection of Clonostachys rosea isolates from Brazilian ecosystems effective in controlling Botrytis cinerea. Biological Control. 34:132 - 143.

PANDEY A. 2003. Solid-state fermentation. Biochemical Engineering Journal. 13:81 - 84 .

PERTOT, I., ZASSO, R., AMSALEM, L., BALDESSARI, M., ANGELI, G. y ELAD, Y., 2008. Integrating biocontrol agents in strawberry powdery mildew control strategies in high tunnel growing systems. Crop Protection. 27:622 - 631 .

ROBERTI, R., VERONESI, A., CESARI, A., CASCONE, A., DI BERARDINO I, BERTINI, L, y CARUSO, C. 2008. Induction of PR proteins and resistance by the biocontrol agent Clonostachys rosea in wheat plants infected with Fusarium culmorum. Plant Science. 175:339 - 347.

RODRÍGUEZ, M.A., CABRERA, G., GOZZO, F.C., EBERLIN, M.N. y GODEAS, A. 2011. Clonostachys rosea BAFC3874 as a Sclerotinia sclerotiorum antagonist: mechanisms involved and potential as a biocontrol agent. Journal of Applied Microbiology. 110:1177 - 1186.

SABARATNAM, S. y TRAQUAIR, J.A. 2002. Formulation of a Streptomyces biocontrol agent for the suppression of Rhizoctonia dampingoff in tomato transplants. Biological Control. $23: 245-253$.

SARAIVA, M.S. 2009. Promoção massal de Clonostachys rosea. Dissertação de Mestrado, Universidade Federal de Viçosa. Viçosa, MG, Brasil.

SAREMBAUD, J.A. y FEINBERG, M. 2007. Comparison of shelf-life appraisal techniques 
for the estimation of the limiting date of stability for agrifood reference materials. Analytica Chimica ACTA. 603:75 - 83.

SCHILLER, C.T. y SINCLAIR, J.B. 1984. Microorganisms associated with soybean vascular exudate and plant parts. International Journal of Tropical Plant Diseases. 2:1 - 4 .

SCHROERS, H.J., SAMUELS, G.J., SEIFERT, K.A. y GAMS, W. 1999. Classification of the mycoparasite Gliocladium roseum in Clonostachys as $C$. rosea, its relationship to Bionectria ochroleuca, and notes on other Gliocladium-like fungi. Mycologia. 91:365 - 385.

SHTIENBERG, D. y ELAD Y. 1997. Incorporation of weather forecasting in integrated, biological-chemical management of Botrytis cinerea. Phytopathology. 87:332 - 340.

STEWART, A. y HARRISON, Y.A. 1989. Mycoparasitism of sclerotia of Sclerotium cepivorum. Australas. Plant Pathology.18:10 - 14.

SUTTON, J.C., LIU, W., MA, J., BROWN, W.G., STEWART, J.F. y WALKER, G.D. 2008. Evaluation of the fungal endophyte Clonostachys rosea as an inoculant to enhance growth, fitness and productivity of crop plants. Acta Horticulturae. 782:279 - 286.

SUTTON, J.C., L.IU, W., HWANG, R. y OWENGOING, N. 2002. Ability of Clonostachys rosea to establish and supress sporulation potencial of Botrytis cinerea in deleafed stems of hydroponic greenhouse tomatoes. Biocontrol Science and Technology. 12:413 - 425.

SUTTON, J.C., LI, D.W., PENG, G., YU, H., ZHANG, P. y VALDEBENITO-SANHUENZA, R.M. 1997. Gliocladium roseum: A versatile adversary of Botrytis cinerea in crops. Plant Disease. $81: 316-328$.
SUTTON J.C. 1995. Evaluation of micro-organisms for biocontrol: Botrytis cinerea and strawberry, a case study. Advances in Plant Pathology Vol. 2, New York, Academic Press. 173-190 p.

SUTTON, J.C. y YU, H. 1994. Inoculum production of Gliocladium roseum. Ontário: Departament of Environmental Biology. University of Guelph, 1p.

SUTTON, J.C. y PENG, G. 1993. Biocontrol of Botrytis cinerea in strawberry leaves. Phytopathology. 83:615 - 621.

TATAGIBA, J.S., MAFFIA, L.A., BARRETO, R.W., ALFENAS, A.C. y SUTTON, J.C. 1998. Biological control of Botrytis cinerea in residues and flowers of rose (Rosa hybrida). Phytoparasitica. 26:8 - 19.

TOLEDO, A.V., VIRLA, E., HUMBER, R.A., PARADELL, S.L. y LASTRA, C.C.L. 2006. First record of Clonostachys rosea (Ascomycota : Hypocreales) as an entomopathogenic fungus of Oncometopia tucumana and Sonesimia grossa (Hemiptera : Cicadellidae) in Argentina. Journal of Invertebrate Pathology. 92:7 - 10.

VALDEBENITO-SANHUENZA,R.M., SUTTON, J.C, PERAZZOLO, I, CZERMAINSKI A.B.C. 1997. Controle Biológico de Botrytis cinerea em morangueiros cultivados em estufa. Fitopatologia Brasileira. 22:69 - 73.

VEGA, F.E., POSADA, F., AIME, M.C., PAVARIPOLL, M., INFANTE, F. y REHNER, A.S. 2008. Entomopathogenic fungal endophytes. Biological Control. 46:72 - 82.

VICCINI G. 2004. Otimização da produção de esporos do fungo Clonostachys rosea - um biopesticida para a cultura do morangueiro. Dissertação de Mestrado. Paraná PR, Brasil. 
VON STOWASSER E.S. y FERREIRA, F.A. 1997. Avaliação de fungos para biocontrole de Botrytis cinerea em viveiros suspensos de Eucalipto. Revista Árvore. 21:147 - 153.

WRAIGHT, S.P., JACKSON, M.A. y KOCK, S.L. 2001. Production, Stabilization and Formulation Biocontrol. In: Butt, T.M.; Jackson, C.W. \& Magan, N. Fungi as Biocontrol Agents: Progress, Problems and Potential. Cab International.

WICKLOW, D.T., ROTH, S., DEYRUP, S.T. y GLOER, J.B. 2005. A protective endophyte of maize: Acremonium zeae antibiotics inhibitory to Aspergillus flavus and Fusarium verticillioides. Mycological Research. 109:610 - 618.

XUE, A.G. 2003. Biological control of pathogens causing root rot complex in field pea using $\mathrm{Clo}-$ nostachys rosea strain ACM941. Phytopathology. 93:329 - 335 .

YU, H. y SUTTON, J.C. 1997. Morphological development and interactions of Gliocladium roseum and Botrytis cinerea in raspberry. Canadian Journal of Plant Pathology. 19:237 - 246.

ZHANG, L., YANG, J.K., NIU, Q.J., ZHAO, X.N., YE, F.P., LIANG, LM. y ZHANG, K.Q. 2008. Investigation on the infection mechanism of the fungus Clonostachys rosea against nematodes using the green fluorescent protein. Applied Microbiology and Biotechnology. 78:983 - 990.

ZOU, C.G., TAO, N., LIU, W.J., YANG, J.K., HUANG, X.W., LIU, X.Y., TU, H.H., GAN Z.W y ZHANG, K.Q. 2010a. Regulation of subtilisin-like protease $\mathrm{prC}$ expression by nematode cuticle in the nematophagous fungus Clonostachys rosea. Environmental Microbiology. 12:3243 - 3252 .
ZOU, C.G., XU, Y.F., LIU, W.J., ZHOU, W., TAO, N., TU, H.H., HUANG, X.W., YANG, J.K. y ZHANG, K.Q. 2010b. Expression of a Serine Protease Gene prC Is Up-Regulated by Oxidative Stress in the Fungus Clonostachys rosea: Implications for Fungal Survival. Plos One. 5(10):1 - 8 . 